\title{
An Active Compliant Control Mode for Interaction with a Pneumatic Soft Robot
}

\author{
J. F. Queißer ${ }^{1}$, K. Neumann ${ }^{1}$, M. Rolf ${ }^{2}$, R. F. Reinhart ${ }^{1}$, and J. J. Steil ${ }^{1}$
}

\begin{abstract}
Bionic soft robots offer exciting perspectives for more flexible and safe physical interaction with the world and humans. Unfortunately, their hardware design often prevents analytical modeling, which in turn is a prerequisite to apply classical automatic control approaches. On the other hand, also modeling by means of learning is hardly feasible due to many degrees of freedom, high-dimensional state spaces and the softness properties like e.g. mechanical elasticity, which cause limited repeatability and complex dynamics. Nevertheless, the realization of basic control modes is important to leverage the potential of soft robots for applications. We therefore propose a hybrid approach combining classical and learning elements for the realization of an interactive control mode for an elastic bionic robot. It superimposes a low-gain feedback control with a feed-forward control based on a learned simplified model of the inverse dynamics which considers only equilibria of the robot's dynamics. We demonstrate on the Bionic Handling Assistant how a respective inverse equilibrium model can be learned and effectively exploited for quick and agile control. In a second step, the control scheme is extended to an active compliant control mode. It implements a kind of gravitation compensation to allow for kinesthetic teaching of the robot based on the implicit knowledge of gravitational and mechanical forces that are encoded in the learned equilibrium model. We finally discuss that this control scheme may be implemented also on other soft robots to provide the avenue towards their applications in general manipulation tasks.
\end{abstract}

\section{INTRODUCTION}

In recent years, an increasing number of soft robots have surfaced in various forms and fields. In their development, researchers have been driven by various motivations. Bioroboticists, for instance, utilize the "understanding by building" approach which is well established in order to complement experimental and theoretical work on biological mechanisms. Prominent examples include artificial salamanders [1], hexapods [2], snakes [3], worms [4], octopus [5], or smaller quadrupeds [6]. In a similar vein, researchers have build humanoid robots like [7], [8], or [9] with the background motivation to understand the role of embodiment in cognition [10] and human-like motor behavior [11]. All the mentioned approaches share the explicit or implicit interest to investigate the interplay of morphology and computation, most prominently phrased under the notion of morphological

${ }^{1}$ J. F. Queißer, K. Neumann, R. F. Reinhart and J. J. Steil are with the Research Institute of Cognition and Robotics - CoR-Lab, Faculty of Technology at Bielefeld University, Universitätsstr. 25, 33615 Bielefeld, Germany, Email: \{jqueisse, kneumann, freinhar, jsteil\}ecor-lab.uni-bielefeld.de, www.cor-lab.de

${ }^{2}$ M. Rolf is with the Emergent Robotics Laboratory, Department of Adaptive Machine Systems at Osaka University, 565-0871 Asada Laboratory, F1-401, 2-1, Yamadaoka, Suita city, Osaka, Japan, Email: matthias@ams.eng.osaka-u.ac.jp, www.er.ams. eng.osaka-u.ac.jp computation [12]. On the other hand, there is an increasing interest from several application fields in soft robotics. From the perspective of safe human-robot interaction, intrinsically safe and fully passive compliant soft-robot platforms like the Bionic Handling Assistant (BHA, [13], see Fig. 1) or components like a soft skin [14] are being developed. Yet another very exciting thread of application-driven research on unusual, flexible and soft mechanism originates from the development of novel micro-invasive medical instruments [15], [16], [17], which have morphologies very similar to that of a worm or the mentioned elephant trunk. Such instruments are ment to bend inside the body when moved towards the hot spot of a surgery. These platforms share the experimental mechanics and actuation designs, where kinematic or dynamic models are hardly available and often have to be approximated, e.g. for the BHA [18]. Typically, standard methods of model-based automatic control cannot be applied easily. But also learning methods are not easy to apply. The main reason is that soft-mechanisms often involve high-dimensional actuation with heavy redundancy, have slow and complex mechanical dynamics often including hysteresis, and exhibit long control delays. Problems for holistic learning approaches thus include that exploration suffers from the curse of dimensionality and simulations are not available again for the lack of models. The generation of training examples from the robot itself is difficult and costly, because the mechanism has to be executed for each sample, and the reproducibility of actions and their results is limited. We have previously shown in [19] that the kinematic control of such robots can be effectively solved

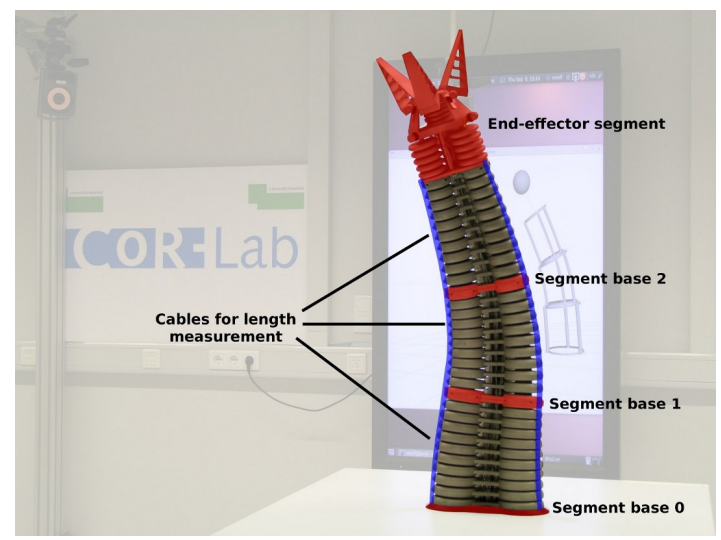

Fig. 1: The Bionic Handling Assistant (BHA) with its segments and the respective length sensors. 
by novel, biological inspired learning schemes that do not rely on exhaustive exploration. However, also lower levels of control pose significant challenges on soft robots, yet being essential to exploit the robots' full potential for safe physical human-robot interaction. While the robot's soft material and actuation permit close spatial proximity between human and robot without posing a threat to the human, its material properties can not be productively utilized of the shelf. It is desirable to be able to freely move the robot to configure its posture [20] or teach in movements to be executed [21]. Typical application scenarios are small scale production lines in which expert programming of the robot is an essential cost factor. In such scenarios, naive users should be able to "program" how a robot executes a task by kinesthetically teaching it. The use of such active compliant control modes have already been shown in industrial contexts [22], but the very control so far required fast and accurate force sensing as well as accurate models of the robot itself, both of which are typically unavailable on soft robots.

In this paper, we argue that to leverage the full potential of soft-robotics for applications we need to resort to a hybrid approach to control, which combines elements from classical automatic control with learning approaches in order to realize novel functions and interactive control modes. While we demonstrate this proposal based on the BHA, we believe that the underlying principle is applicable to other robots as well. More specifically, we show in this paper that a classical approach to deal with long delays by superimposing a model of the inverse dynamics with a standard feedback control [23] is highly useful on the BHA, despite the fact that we have to substitute the inverse dynamics model in the feed-forward path by a learned inverse equilibrium model. This combination provides more efficient position control on the BHA, which otherwise suffers from long delays due to its low-pressure actuation in combination with its elastic mechanical hardware. Furthermore, we show that the same control scheme can be used to implement an actively controlled deformation mode that adds to the mechanical compliance and allows the BHA to follow externally applied forces to enable kinesthetic teaching. While such control modes usually require precise force sensing and full and precise models (both of which are unavailable on the BHA), it turns out that with the BHA's elastic mechanics, an interactive control mode can be achieved without such prerequisites. The paper is structured as follows: Sec. II describes the Bionic Handling Assistant. The proposed control scheme is introduced in Sec. III. Sec. IV presents the data-driven equilibrium model of the BHA including the description of the data-set, the learning algorithm used to estimate the equilibrium model, and the impact of the model on the control of the robot. Sec. VI exposes the proposed interaction method in a human-robot interaction scenario.

\section{The Bionic Handling Assistant (BHA)}

The BHA [13], [24] is a pneumatically actuated, awardwinning [25] robot platform manufactured by Festo and inspired by elephant-trunks. It belongs to a new class of soft and lightweight robots with pneumatic chambers, which allow for a safe physical interaction between robots and humans. The actuation principle with several segments of continuous and parallel components has gathered increasing interest in robotics research over the last decade. The actuators operate with low-pressure pneumatics, which is not sufficient for a reliable control of the robot: air pressure only describes a force. The structure of the BHA is separated into three segments (see Fig. 1). Each segment consists of three triangular arranged air chambers. Therefore the main flexibility of the BHA is based on 9 air chambers that extend their length in relation to the pressure in those chambers. A fourth end-effector segment is also available but was neglected for this work. An active depression of the pressure of the chambers is not possible, solely the tension of the extended body reforms the structure back to the home position. The robot has no fixed joint angles, each robot segment starts to bend in the case that the three chambers reach different lengths. Beside pressure sensors that are included in the air valves, the BHA is equipped with cable potentiometers that allow to measure the outer length of the air chambers providing geometric information about the robot shape. The segments of the BHA together with the attached cable length sensors are depicted in Fig. 1. In the following, we consider the pneumatic actuators of each segment separately and ignore the influence from the other segments. This approximation is reasonable because the inter-segment interaction is neglectable in comparison to the intra-segment interaction due to robot's morphology and its light weight.

\section{INVERSE EQUILIBRIUM MODEL FOR LENGTH CONTROL}

A reliable and fast controller of the air chamber lengths is an indispensable prerequisite for the application of the BHA. The control can, in principle, be done with standard schemes like proportional integral derivative control (PID). The fundamental problem is that these approaches rely on quick and reliable feedback from the robot, while the BHA only provides very delayed and noisy feedback due to its pneumatic actuation and the visco-elastic mechanics. Consequently, the PID control can only be applied with low gains, which corresponds to slow movements.

An inverse dynamics model $\mathbf{f}$ of the robot operating as feed-forward controller in addition to the low-gain feedback control could significantly decrease control delays. For the BHA, such an inverse model would map actuator lengths 1 and their derivatives $\mathbf{i}$ and $\ddot{\mathbf{l}}$ to pressures $\mathbf{p}$ in the actuators.

$$
\mathbf{p}(t)=\mathbf{f}(\mathbf{l}(t), \mathbf{i}(t), \ddot{\mathbf{l}}(t))
$$

However, the downside of the biologically inspired design of the BHA is that hardly any analytic models are available. Traditional approaches such as inverse dynamics based control becomes intractable. This fact qualifies learning as an essential tool for modeling, but collecting a data set that fully represents the inverse dynamics of the BHA is difficult. 
We therefore consider a simplified model in this paper, which is restricted to the mechanical equilibrium points $\mathbf{l}^{*}$ of the robot's dynamics. Equilibrium points are achieved by applying a constant pressure $\mathbf{p}^{*}$ until convergence of the lengths for a single segment. In such a state, neither lengths nor pressures of the pneumatic actuators change over time: $\dot{\mathbf{p}}=\mathbf{i}=\ddot{\mathbf{l}}=0$. The formulation of the inverse dynamics in Eq. (1) thus simplifies to the following:

$$
\mathbf{p}^{*}=\mathbf{f}\left(\mathbf{l}^{*}, 0,0\right) \Leftrightarrow \hat{\mathbf{p}}\left(\mathbf{l}^{*}\right)=\mathbf{p}^{*},
$$

where $\hat{\mathbf{p}}$ denotes the inverse equilibrium model that represents the direct relation between length $\mathbf{l}^{*}$ and pressures $\mathbf{p}^{*}$. The inverse equilibrium model provides a direct estimation of a reasonable control signal and can therefore serve as a feedforward control signal that is applied immediately without waiting for delayed feedback.

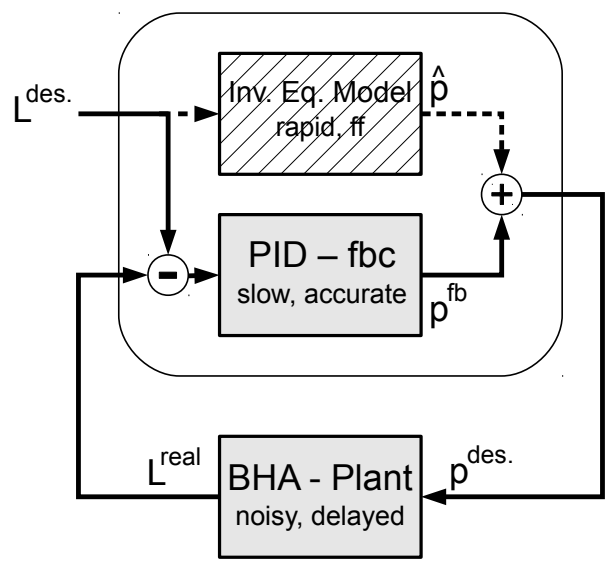

Fig. 2: The control loop: combination of a learned inverse equilibrium model and a feedback controller. Leads to a fast estimation of the pressure configuration $p^{\text {des }}$ for the chamber lengths $L^{\text {des }}$.

A schematic illustration of this approach is shown in Fig. 2. The image visualizes the BHA plant with its noisy and delayed feedback, the PID feedback controller, and the inverse equilibrium model. The BHA receives pressure commands, which are computed by a superposition of the low-gain PID controller and the feed-forward control signal from the inverse equilibrium model. The feed-forward controller computes pressures from desired length values by means of the inverse equilibrium model. PID control is based on the difference of the desired length values and the sensed length values. The PID controller thereby corrects the errors of the feed-forward controller in the feedback loop.

Learning the inverse equilibrium model from scratch is nevertheless difficult for several reasons: first, the underlying dynamics of the BHA result in a non-linear behavior which requires a model with appropriate complexity in order to capture the structure of the data to a sufficient degree. Second, data sampling is limited because the time until the physical deformations of the robot have reached a mechanical equilibrium can take up to 20 seconds for a single data point. Third, the resulting samples are very noisy due to physical hysteresis effects induced by the visco-elasticity of the robot's soft material. Finally, the material changes its properties due to the history of the manipulation. A predefined working area can change over time. Well-behaved extrapolation is thus a strong requirement on a learned inverse equilibrium model. Machine learning approaches which are trained on such data without additional efforts are prone to overfitting. To cope with these issues thus becomes an important requirement for the learner. We apply an approach that uses prior knowledge about the physical behavior of the BHA in order to derive a reasonable model of the length-to-pressures relation in a mechanical equilibrium in a data-driven manner [26]. Note, that any other algorithm that can handle these requirements is potentially applicable.

\section{LEARNING THE BHA'S INVERSE EQUILIBRIUM MODEL WITH PRIOR KNOWLEDGE}

For learning of an inverse equilibrium model, we apply a machine learning approach that is able to incorporate prior knowledge about physical constraints of the BHA in order to reduce over-fitting from few and noisy data and achieve wellbehaved extrapolation. The following prior knowledge about the BHA is considered: (i) maximum and minimum pressure of the actuators are known in advance, and (ii) the groundtruth behavior per axis is strictly monotonous, because higher pressure in one actuator physically leads to an extension of this actuator.

Further, the observation that the entire mapping from length sensor values to chamber pressures can be separated into three, independent problems is used. This means that one inverse equilibrium model per segment (see Fig. 1) is learned, which significantly reduces the demand for training data. This assumption neglects the gravity effects caused by a deflection of the remaining segments. However, these effects are rather small due to the robot's light weight and are corrected by the feedback controller.

The applied learning scheme is called Constrained Extreme Learning Machine (CELM) and was first introduced in [26]. It is a feed-forward neural structure that comprises three layers of neurons. For the inverse equilibrium model of a single segment of the BHA, the CELM comprises $\mathbf{l} \in \mathbb{R}^{I=3}$ input, $\mathbf{h} \in \mathbb{R}^{R}$ hidden, and $\hat{\mathbf{p}} \in \mathbb{R}^{O=3}$ output neurons. The input is connected to the hidden layer by the input matrix $W^{\text {inp }} \in \mathbb{R}^{R \times I}$. The read-out matrix is given by $W^{\text {out }} \in$ $\mathbb{R}^{O \times R}$. For input $\mathbf{l}$, the output of neuron $i$ is computed by

$$
\hat{\mathbf{p}}_{i}(\mathbf{x})=\sum_{j=1}^{R} W_{i j}^{\mathbf{o u t}} f\left(\sum_{k=1}^{I} W_{j k}^{\mathbf{i n p}} x_{k}+b_{j}\right),
$$

where $b_{j}$ is the bias for neuron $j$, and $f(x)=\left(1+e^{-x}\right)^{-1}$ the logistic activation function. The components of the input matrix $W^{\text {inp }}$ and the biases $b_{j}$ are drawn from a random distribution and remain fixed after initialization.

Let $\mathcal{D}=(L, P)=\left(\mathbf{l}^{k}, \mathbf{p}^{k}\right)$ with $k=1 \ldots N_{\text {tr }}$ be the data set for training, where $N_{\text {tr }}$ is the number of training samples. $L \in \mathbb{R}^{I \times N_{\text {tr }}}$ is the collection of lengths, and $P \in \mathbb{R}^{O \times N_{\text {tr }}}$ is the matrix of target pressures for all $N_{\text {tr }}$ samples. Supervised 
learning is restricted to the read-out weights $W^{\text {out }}$ and accomplished by solving a quadratic program which is subject to condition (i) and (ii) rephrased as linear constraints:

$$
\left\|W^{\text {out }} \cdot H(L)-P\right\|^{2}+\alpha \cdot\left\|W^{\text {out }}\right\|^{2} \rightarrow \min
$$

subject to:

$$
\text { (ii) } p_{\min }^{s}<\hat{p}_{i}(\mathbf{l})<p_{\max }^{s}: \forall \mathbf{l} \in \Omega,
$$

where $H(L) \in \mathbb{R}^{R \times N_{\text {tr }}}$ is the matrix collecting the hiddenlayer states. The growth of the read-out weights is controlled by the regularization parameter $\alpha . \Omega$ is a predefined region in the model's input space, and $s, i=1,2,3$ denote the segment and the output and input dimension.

The prior knowledge given by (i), (ii) defines inequalities on the learning parameters $W^{\text {out }}$ at specific points $\mathbf{l}^{\prime} \in \Omega$, which are sampled according to the approach in [26]. Note that these inequalities are linear in $W^{\text {out }}$. It was shown in [26] that a well-chosen sampling of the points $\mathbf{l}^{\prime}$ is sufficient for generalization of the point-wise constraints to the continuous region $\Omega$.

\section{Experimental Evaluation of LEngth Control}

This section contains the results of a cross-validation test and the experimental evaluation of a linear model and the CELM model when applied in parallel to the PID feedback controller.

\section{A. BHA Data Set}

For training of inverse equilibrium models, a data set is recorded. It captures the relation between the geometric length of the air chambers for each segment and the corresponding pressures in a mechanical equilibrium. Pressures are measured in milli-bar and the segment lengths in meters. For each segment, the pressure space is explored by applying pressures between minimum and maximum value in five equidistant steps. This results in a pressure grid comprising $5 \times 5 \times 5=125$ samples. For each pressure, the resulting combination of three lengths was recorded after a waiting phase of 20 seconds in order to reach the mechanical equilibrium. In order to deal with the inherent variation due to the visco-elastic material, this process is repeated five times with different traversal orderings, such that 625 samples per segment are available for learning. The minimum and maximum pressures, and the resulting length ranges are collected in TABLE I.

TABLE I: Properties of the BHA data set. Including pressure and length ranges of the segments of the actuator.

\begin{tabular}{c|c|c|c|c|c|c} 
Seg. & $p_{\max }$ & $p_{\min }$ & $l_{\max }$ & $l_{\min }$ & $\mathrm{N}$ & \#Trials \\
\hline 1 & $800 \mathrm{mbar}$ & $0 \mathrm{mbar}$ & $0.32 \mathrm{~m}$ & $0.17 \mathrm{~m}$ & 625 & 5 \\
2 & $1000 \mathrm{mbar}$ & $0 \mathrm{mbar}$ & $0.33 \mathrm{~m}$ & $0.16 \mathrm{~m}$ & 625 & 5 \\
3 & $1200 \mathrm{mbar}$ & $0 \mathrm{mbar}$ & $0.32 \mathrm{~m}$ & $0.16 \mathrm{~m}$ & 625 & 5
\end{tabular}

The grid for the applied pressures of segment 1 is illustrated in Fig. 3 (first). The corresponding length values recorded
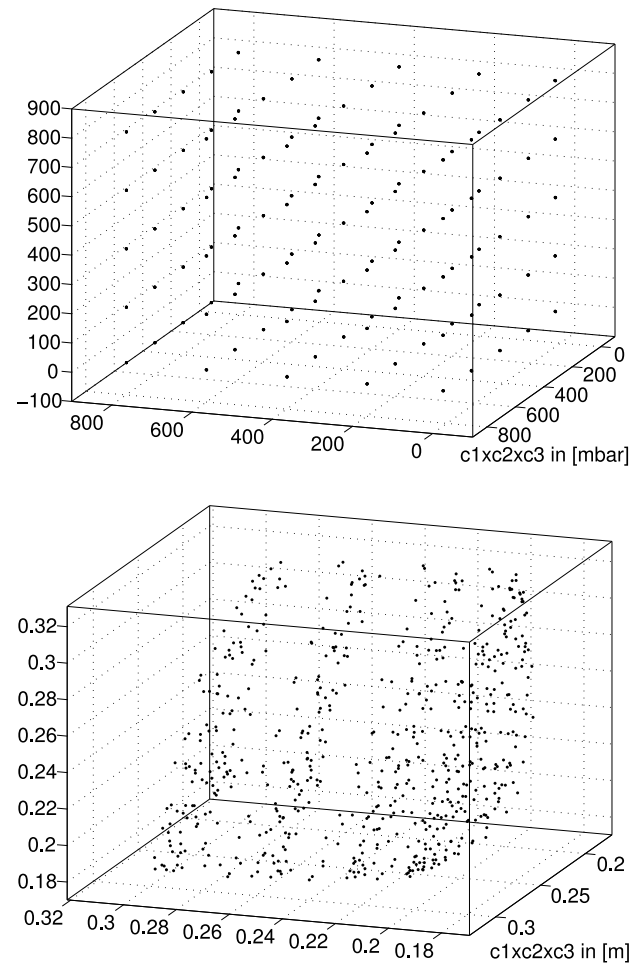

Fig. 3: Data set for chambers 1-3 of segment 1, each dimension represents one chamber. Pressure grid with five samples per dimension (top) and the corresponding length values (bottom). The non-linear relation between lengths and pressures leads to gaps in the input space of the data.

on the robot are shown in Fig. 3 (second). The data are nonlinear, with huge gaps in the middle part of the target data, for which generalization is critical.

\section{B. Cross-Validation and Generalization}

The generalization performance of the learned models is evaluated on the BHA data set by cross-validation. Linear models (LM)

$$
\hat{\mathbf{p}}(\mathbf{l})=W^{T} \mathbf{l}+\mathbf{b}
$$

trained by linear regression and the constrained ELM model (CELM) with additional use of prior knowledge are compared. An appropriate error measure for the learned inverse equilibrium models is the per-axis average-deviation from the measured ground truth value:

$$
E=\frac{1}{N} \sum_{k=1}^{N} \frac{1}{D} \sum_{d=1}^{D}\left\|p_{d}^{k}-\hat{p}_{d}\left(\mathbf{l}^{k}\right)\right\|,
$$

where $N$ is the number of samples and $D=3$ is the input and output dimensionality.

The results shown in TABLE II are obtained by crossvalidation over the five trials measured by the error function in Eq. (6). For each fold, four trials are used for training and one trial is used for testing the generalization ability of the models. Afterwards, the errors are averaged over the five folds. We state mean and standard deviation over the different 
TABLE II: Cross-validation errors. Comparison between linear model (LM) and constrained ELM (CELM).

\begin{tabular}{c|c|c} 
Segment & LM (tr / te) $[\mathrm{mbar}]$ & CELM \\
\hline 1 & $48.9 \pm 0.8 / 52.7 \pm 4.8$ & $27.8 \pm 1.7 / 36.5 \pm 7.6$ \\
2 & $74.9 \pm 3.1 / 83.0 \pm 13.9$ & $46.0 \pm 2.9 / 61.7 \pm 11.0$ \\
3 & $74.7 \pm 0.6 / 78.4 \pm 5.2$ & $41.4+-1.6 / 54.6+-5.3$
\end{tabular}

cross-validation folds in TABLE II. The mapping ability of the LM is too poor to capture the structure encoded in the BHA data - the training (tr) and test (te) errors are large and indicate under-fitting. The CELM, in contrast, performs significantly better and is able to capture the non-linearity of the mapping underlying the data.

\section{Experiment-In-The-Loop}

Experiments on the robot show the benefits of the learned inverse equilibrium model on the length control. For a quantitative comparison, we measure the time until convergence of the lengths to different target values. Convergence is achieved after the desired lengths are reached with a certain accuracy $\varepsilon$ as illustrated in Fig. 4:

Given the target lengths $\hat{\mathbf{l}}$ and the measured lengths $\mathrm{l}$, convergence is reached at time $\tilde{t}$ if the error $\left\|\hat{l}_{i}(t)-l_{i}(t)\right\|$ for all actuators $i$ is below the threshold $\varepsilon$ for all time steps after $\tilde{t}$ until $t_{\text {end }}$ is reached. For the experiments in this section $t_{\text {end }}=10$ seconds was selected.

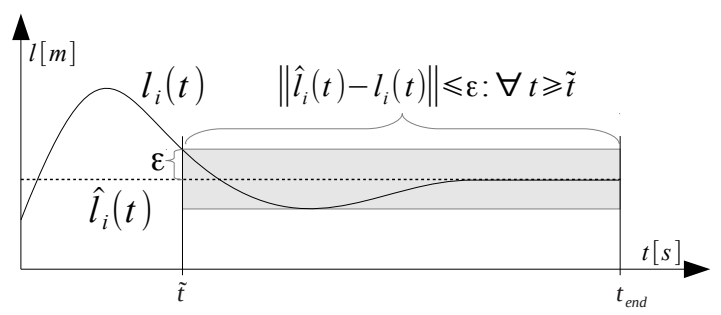

Fig. 4: Illustration of the convergence time measure. Convergence time is defined as the timespan $\tilde{t}$ whereupon the deviation of the measured lengths $l_{i}(t)$ of the chambers and the target lengths $\hat{l}_{i}(t)$ stays below the threshold $\varepsilon$ until $t_{\text {end }}$ is reached.

The convergence time was measured for each tested model on five random postures and repeated for ten times. Fig. 5 shows the mean convergence time for all trials. It is demonstrated that the length control without a feed-forward control signal, i.e. $\hat{\mathbf{p}}=\mathbf{0}$, requires a much longer convergence time than a feed-forward control with the linear model or the CELM. Furthermore the figure shows that the CELM model benefits from its capabilities to model non-linear data distributions in comparison to the linear model. It allows a more accurate prediction and thus a faster convergence in $68.25 \%$ of all sample postures for all tube sizes and in $82.22 \%$ for a tube size of $\epsilon=0.35 \mathrm{~cm}$.

\section{Equilibrium Model For An ACTIVE COMPliant Control Mode}

In comparison to the implementation of a kinesthetic teaching mode on stiff robots [27], a flexible robot structure allows

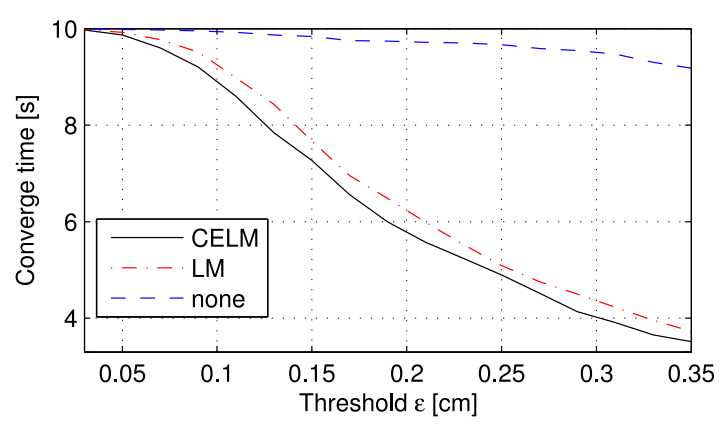

Fig. 5: Convergence time of the length controller using different inverse equilibrium models: ELM with constraints (CELM), linear model (LM) and without inverse equilibrium model (none). Results are shown in relation to the tube size $\epsilon$.

the deformation of the actuator to a certain extend due to its softness. The detection of a deformation, e.g. caused by a human tutor, can be utilized to initiate a modification of the control variables such that the robot complies with the deformed configuration. The learned inverse equilibrium model of the robot can be used to detect deflections from the equilibrium by comparing the measured pressures of the chambers with the expected chamber pressures for the current lengths computed by the inverse equilibrium model. The control target lengths are then adopted such that the current configuration becomes the new equilibrium point of the robot. This morphology-driven external force detection principle reduces the required computational effort and control complexity in comparison to classical approaches based on a full inverse dynamics model and accurate force sensing. Fig. 6 shows the interconnection of the different control modules to enable an active compliant control mode for the BHA. Essentially, the comparison between measured pressure in the

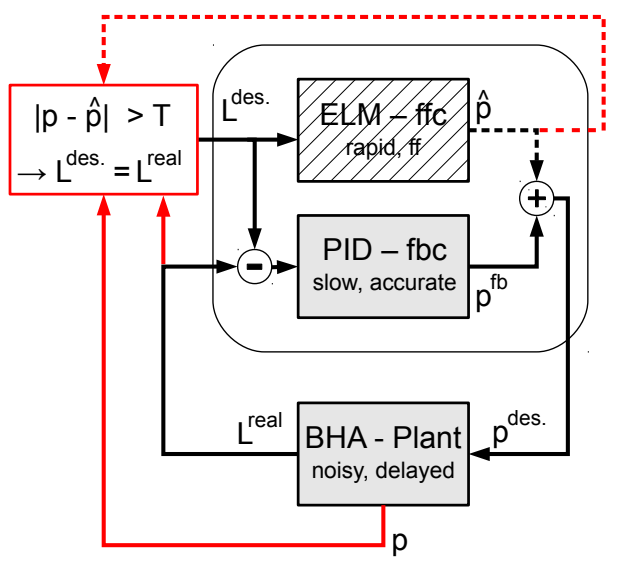

Fig. 6: Active compliant control mode of the BHA achieved by application of a learned inverse equilibrium model of the pressure-to-length relation in a mechanical equilibrium.

pneumatic chambers $\mathbf{p}$ and the predicted pressures according to the learned inverse equilibrium model $\hat{\mathbf{p}}$ is added to the previous control scheme shown in Fig. 2. Whenever the error $\|\mathbf{p}-\hat{\mathbf{p}}\|$ is above a predefined threshold $T$, the set-point 
$L^{\text {des }}$ of the length control system is defined as the measured length sensor values $L^{\text {real }}$. This leads to a redefinition of the set-point if the BHA is deflected from a mechanical equilibrium state. Such a deflection can be induced by a human interacting with the BHA and allows to deform the robot easily in an intuitive manner.

A critical parameter for the functionality and the sensitivity of the external force detection is the threshold $T$. While smaller thresholds can result in drifts of the actuator due to inaccuracies of the inverse equilibrium model, larger thresholds limit the interaction quality due to a improper detection of external forces of the system.

\section{A. Estimation of Threshold T}

In order to obtain a reasonable threshold $T$, we recorded a data set of 25 postures and the respective prediction error values. Four postures of the data set and the corresponding errors are exemplary shown in Fig. 7. The average error of
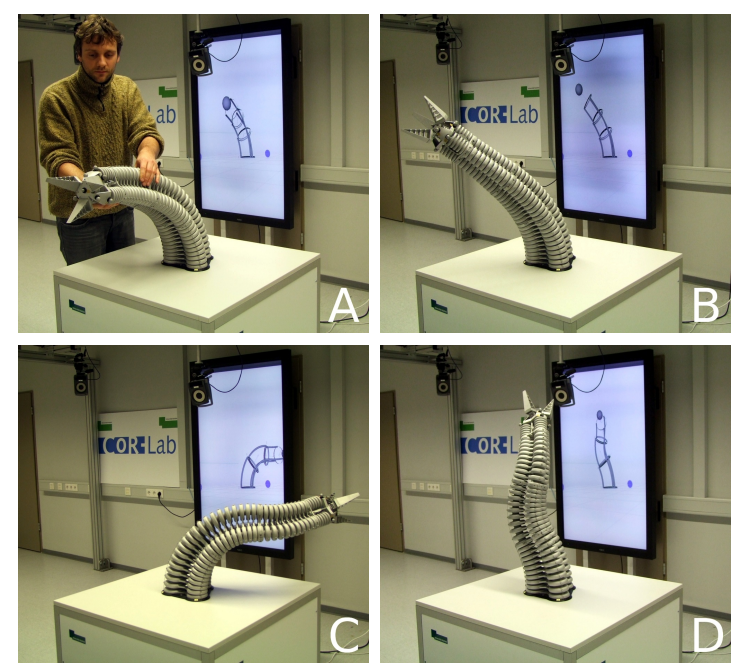

Fig. 7: Stable postures of the BHA after manual reconfiguration in active compliance mode. Manual reconfiguration of the BHA by a human tutor (A). Three exemplary postures from the test data set (B, C and D). Model Errors in the mechanical equilibrium: $50.6 \mathrm{mbar}(\mathrm{B}), 36.6 \mathrm{mbar}(\mathrm{C})$, and 55.9 mbar (D).

the learned inverse equilibrium model is $44.4 \mathrm{mbar}$, while the standard deviation is 8.9 mbar. The maximum and minimum error amounts to $58.7 \mathrm{mbar}$ and $29.1 \mathrm{mbar}$, respectively. This motivates a threshold of $T=60$ mbar.

\section{B. Active Posture Control in Human-Robot Interaction}

Fig. 8 shows a sequence of two manual reconfigurations of the BHA by a human tutor. The start configuration of the robot trunk is relaxed, the pneumatic actuators are deflated. After roughly eleven seconds, the human operator starts to push the robot to the right side which deflects the robot's length and pressure state from the mechanical equilibrium point. This instantly induces an increasing prediction error $\|\mathbf{p}-\hat{\mathbf{p}}\|$ of the learned model $\hat{\mathbf{p}}$. When the error exceeds the threshold $T$, the set point of the desired length is reset to the current length sensor values. The length controller then adopts the pressures accordingly such that the current robot configuration becomes the new equilibrium point of the system. This tracking of the robot posture enables the user to easily change the posture of the robot trunk.

After a short time span, the robot again reached a mechanical equilibrium such that the error falls below the threshold (after approx. 16 seconds). During this time, the arm stays fixed until a second manipulation phase is started by the user (after 45 seconds). The manipulations lasts for five seconds and ends after the desired end posture is reached. The BHA stays stably in this position. This shows that the proposed control scheme is able to provide a useful interactive control mode without the need of complex internal models of the actuator. This human-robot interaction mode offers new fields of application for soft robots in research and practical applications.

\section{DISCUSSION}

In this contribution, we have shown how to enable human robot interaction by utilizing a classical control scheme in combination with a learned approximation of the inverse dynamics that only respects the equilibrium states. The hybrid control architecture significantly decreases control delays by adding the learned inverse equilibrium model in a feed-forward control path to a slow, low-gain feedback controller. While we used the Bionic Handling Assistant, the scheme could as well be applied on other soft robots, if three conditions are met: (i) the robot has mechanical elasticity; (ii) the mechanism is modular in the sense that it can be decomposed in several parts (here segments) that allows for a well posed learning of the inverse equilibrium model under neglection of the interactions with other parts; (iii) the actuation is back-drivable. We believe that this could also be the case for other soft robots, the crucial issue being the decomposition to make the learning feasible. Further, we believe that from the interplay of softness properties, control, and learning more may be discovered in future work. For instance by utilizing the recently very successful methods from reinforcement and explorative learning that use kinesthetic teaching for seeding a subsequent optimization towards higher level goals. The presented results are encouraging towards such applications.

\section{ACKNOWLEDGMENTS}

This research was funded by FP7 under GA. No. 248311-AMARSi, the DFG, Cluster of Excellence 277 "Cognitive Interaction Technology" and the German BMBF within the Intelligent Technical Systems Leading-Edge Cluster.

\section{REFERENCES}

[1] A. Ijspeert, A. Crespi, and J.-M. Cabelguen, "Simulation and robotics studies of salamander locomotion: applying neurobiological principles to the control of locomotion in robots." Neuroinformatics, vol. 3, no. 3, pp. 171-195, 2005.

[2] M. Schilling, J. Paskarbeit, T. Hoinville, A. Hüffmeier, A. Schneider, J. Schmitz, and H. Cruse, "A hexapod walker using a heterarchical architecture for action selection," Front. in Computational Neuroscience, vol. 7, 2013. 

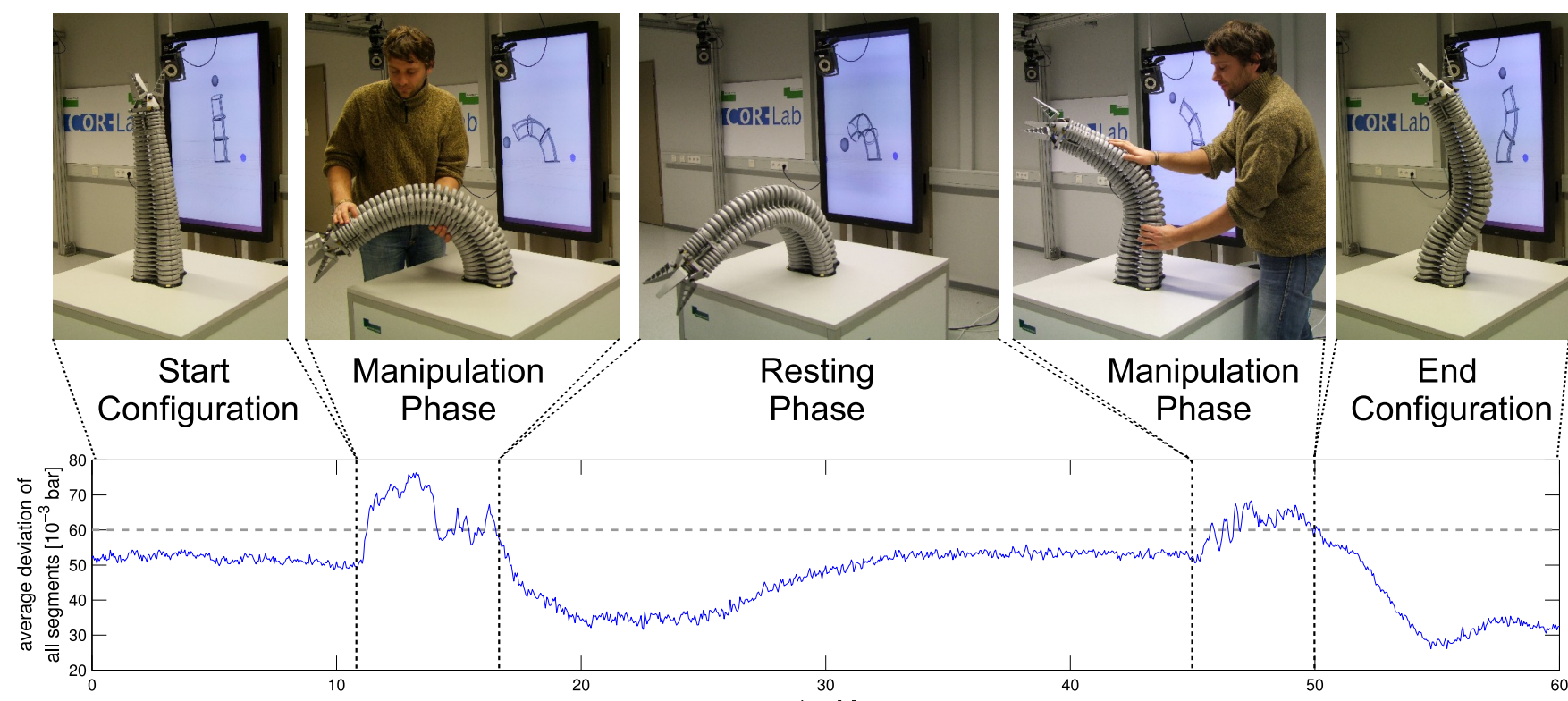

Fig. 8: Active posture control in human-robot interaction. The graph on the lower part of the figure shows the prediction error $\|\mathbf{p}-\hat{\mathbf{p}}\|$ during human-robot interaction. The dashed line marks the selected threshold $T$. It is demonstrated that the prediction error exceeds $T$ during the manipulation phase (adaptation) and falls below $T$ during the resting phase (hold posture).

[3] A. Transeth, R. Leine, C. Glocker, K. Pettersen, and P. Liljeback, "Snake robot obstacle-aided locomotion: Modeling, simulations, and experiments," IEEE Trans. Robotics, vol. 24, no. 1, pp. 88-104, 2008.

[4] S. Seok, C. Onal, K.-J. Cho, R. Wood, D. Rus, and S. Kim, "Meshworm: A peristaltic soft robot with antagonistic nickel titanium coil actuators," IEEE Trans. Mechatronics, vol. 18, no. 5, pp. 1485-1497, 2013.

[5] M. Calisti, M. Giorelli, G. Levy, B. Mazzolai, B. Hochner, C. Laschi, and P. Dario, "An octopus-bioinspired solution to movement and manipulation for soft robots," Bioinspiration \& Biomimetics, vol. 6, no. 3, 2011.

[6] A. Spröwitz, A. Tuleu, M. Vespignani, M. Ajallooeian, E. Badri, and A. Ijspeert, "Towards dynamic trot gait locomotion: Design, control, and experiments with cheetah-cub, a compliant quadruped robot," Int. Journal of Robotics Research, vol. 32, no. 8, pp. 932-950, 2013.

[7] H. Marques, M. Jäntsch, S. Wittmeier, O. Holland, C. Alessandro, A. Diamond, M. Lungarella, and R. Knight, "Ecce1: The first of a series of anthropomimetic musculoskeletal upper torsos," in Int. Conf. on Humanoid Robots, 2010, pp. 391-396.

[8] T. Shirai, J. Urata, Y. Nakanishi, K. Okada, and M. Inaba, "Whole body adapting behavior with muscle level stiffness control of tendondriven multijoint robot," in IEEE Int. Conf. Robotics and Biomimetics, 2011, pp. 2229-2234.

[9] C. Ott, B. Henze, and D. Lee, "Kinesthetic teaching of humanoid motion based on whole-body compliance control with interactionaware balancing," in IROS, 2013, pp. 4615-4621.

[10] R. Pfeifer and J. C. Bongard, How the Body Shapes the Way We Think: A New View of Intelligence (Bradford Books). The MIT Press, 2006.

[11] N. G. Tsagarakis, M. Laffranchi, B. Vanderborght, and D. Caldwell, "A compact soft actuator unit for small scale human friendly robots," in IEEE Int. Conf. on Robotics and Automation, 2009, pp. 4356-4362.

[12] H. Hauser, A. J. Ijspeert, R. M. Füchslin, R. Pfeifer, and W. Maass, "Towards a theoretical foundation for morphological computation with compliant bodies," Biological Cybernetics, vol. 105, no. 5-6, pp. 355370, 2011.

[13] A. Grzesiak, R. Becker, and A. Verl, "The Bionic Handling Assistant - A Success Story of Additive Manufacturing," Assembly Automation, vol. 31, no. 4, pp. 329-333, 2011.

[14] V. Duchaine, N. Lauzier, M. Baril, M.-A. Lacasse, and C. Gosselin, "A flexible robot skin for safe physical human robot interaction," in IEEE Int. Conf. on Robotics and Automation, 2009, pp. 3676-3681.

[15] D. Roppenecker, A. Pfaff, J. Coy, and T. Lueth, "Multi arm snake-like robot kinematics," in IEEE/RSJ Int. Conference on Intelligent Robots and Systems, 2013, pp. 5040-5045.

[16] K.-W. Kwok, K. H. Tsoi, V. Vitiello, J. Clark, G. Chow, W. Luk, and G.-Z. Yang, "Dimensionality reduction in controlling articulated snake robot for endoscopy under dynamic active constraints," IEEE Trans. on Robotics, vol. 29, no. 1, pp. 15-31, 2013.

[17] A. Jiang, E. Secco, H. Wurdemann, T. Nanayakkara, P. Dasgupta, and K. Athoefer, "Stiffness-controllable octopus-like robot arm for minimally invasive surgery," in Workshop on New Technologies for Computer/Robot Assisted Surgery (CRAS 2013), 2005.

[18] M. Rolf and J. Steil, "Constant curvature continuum kinematics as fast approximate model for the bionic handling assistant," in IEEE/RSJ Int. Conf. Intelligent Robots and Systems, 2012, pp. 3440-3446.

[19] _ _ "Efficient exploratory learning of inverse kinematics on a bionic elephant trunk," IEEE Transactions on Neural Networks and Learning Systems, vol. 25, no. 6, pp. 1147-1160, 2014.

[20] A. Lemme, A. Freire, G. Barreto, and J. Steil, "Kinesthetic teaching of visuomotor coordination for pointing by the humanoid robot icub," Neurocomputing, vol. 112, pp. 179-188, 2013.

[21] B. Akgun, M. Cakmak, J. W. Yoo, and A. L. Thomaz, "Trajectories and keyframes for kinesthetic teaching: A human-robot interaction perspective," in ACM/IEEE HRI, 2012, pp. 391-398.

[22] S. Wrede, C. Emmerich, R. Grünberg, A. Nordmann, A. Swadzba, and J. Steil, "A user study on kinesthetic teaching of redundant robots in task and configuration space," Journal of Human-Robot Interaction, vol. 2, pp. 56-81, 2013.

[23] J. Slotine and W. Li, Applied Nonlinear Control. Prentice Hall, 1991.

[24] K. J. Korane, "Robot imitates nature," Machine Design, vol. 82, no. 18, pp. 68-70, 2010.

[25] Deutscher Zukunftspreis (German Future-Award), 2010. [Online]. Available: http://www.deutscher-zukunftspreis.de/en/content/2010

[26] K. Neumann, M. Rolf, and J. J. Steil, "Reliable integration of continuous constraints into extreme learning machines," Journal of Uncertainty, Fuzziness and Knowledge-Based Systems, vol. 21, pp. 35-50, 2013.

[27] W. Wang, R. N. Loh, and E. Y. Gu, "Passive compliance versus active compliance in robot-based automated assembly systems," Industrial Robot: An International Journal, vol. 25, no. 1, pp. 48-57, 1998. 